\title{
Nut consumption is associated with better nutrient intakes: results from the 2008/09 New Zealand Adult Nutrition Survey
}

\author{
Rachel C. Brown ${ }^{1 *}$, Siew Ling Tey ${ }^{2}$, Andrew R. Gray ${ }^{3}$, Alex Chisholm ${ }^{1}$, Claire Smith ${ }^{1}$, Elizabeth Fleming ${ }^{1}$ \\ and Winsome Parnell ${ }^{1}$ \\ ${ }^{1}$ Department of Human Nutrition, University of Otago, PO Box 56, Dunedin 9054, New Zealand \\ ${ }^{2}$ Clinical Nutrition Research Centre, Singapore Institute for Clinical Sciences, A*STAR, 14 Medical Drive, \#07-02, Singapore \\ 117599, Singapore \\ ${ }^{3}$ Department of Preventive and Social Medicine, University of Otago, PO Box 56, Dunedin 9054, New Zealand \\ (Submitted 8 June 2015 - Final revision received 12 August 2015 - Accepted 15 September 2015 - First published online 20 October 2015)
}

\section{Abstract}

A limited number of studies have examined associations between nut consumption and nutrient intakes or diet quality. None has investigated these associations in the Southern Hemisphere. The purpose of this study was to examine associations between nut consumption and nutrient intakes among adult New Zealanders. Data from the 24-h recalls of 4721 participants from the cross-sectional 2008/09 New Zealand Adult Nutrition Survey (2008/09 NZANS) were used to determine whole nut intake and total nut intake from all sources as well as nutrient intakes. Regression models, both unadjusted and adjusted for potential confounders, were used to estimate differences in nutrient intakes between those consuming and those not consuming nuts. From adjusted models, compared with non-whole nut consumers, whole nut consumers had higher intakes of energy and percentage of energy from total fat, MUFA and PUFA, whereas percentage of energy from SFA and carbohydrate was lower (all $P \leq 0.025$ ). After the additional adjustment for energy intake, whole nut consumers had higher intakes of dietary fibre, vitamin $\mathrm{E}$, folate, $\mathrm{Cu}, \mathrm{Mg}, \mathrm{K}, \mathrm{P}$ and $\mathrm{Zn}$ (all $P \leq 0 \cdot 044$ ), whereas cholesterol and vitamin $\mathrm{B}_{12}$ intakes were significantly lower (both $P \leq 0 \cdot 013$ ). Total nut consumption was associated with similar nutrient profiles as observed in whole nut consumers, albeit less pronounced. Nut consumption was associated with better nutrient profiles, especially a lower intake of SFA and higher intakes of unsaturated fats and a number of vitamins and minerals that could collectively reduce the risk for chronic disease, in particular for CVD.

Key words: Nut intake: Nutrient intakes: Population surveys: CVD

Nuts are rich in unsaturated fatty acids, vitamins, minerals and bioactive compounds, which collectively are likely to contribute to their well-recognised health properties, in particular to reduce CVD risk ${ }^{(1-3)}$. If consumed in sufficient amounts, the nutrients present in nuts are likely to improve diet quality, which should translate into overall improved health outcomes for nut consumers compared with non-nut consumers.

Although individual nuts differ in types and amounts of nutrients, all nuts are considered to be nutrient dense ${ }^{(3,4)}$. Nuts are generally rich sources of cis-unsaturated fatty acids, fibre and plant protein including arginine ${ }^{(3,5)}$. In addition, individual nuts can contribute important amounts of folate, vitamin E, Ca, $\mathrm{Mg}, \mathrm{Cu}, \mathrm{Zn}$, Se and K. Nuts also contain bioactive substances such as phytosterols, antioxidants and phenolic compounds, which are further likely to contribute positively to health outcomes $^{(6)}$. Thus, frequent nut consumption is likely to make important contributions towards a healthy diet. Indeed
O'Neil et al. ${ }^{(7)}$ showed that tree nut consumption improved nutrient intake and diet quality in the National Health and Nutrition Examination Survey (NHANES) 1999-2004 cohort. In particular, the diets of tree nut consumers contained greater amounts of dietary fibre, vitamin $\mathrm{E}, \mathrm{Ca}, \mathrm{Mg}$ and $\mathrm{K}$ and lower amounts of $\mathrm{Na}$ compared with non-consumers. Using the Healthy Eating Index-2005, diet quality was found to be higher in nut consumers. This was also seen in their analysis of the NHANES 2005-2010 cohort $^{(8)}$. In addition, this group found similar improvements in diet quality when assessing 'out-of-hand' tree nut consumers $^{(9)}$. 'Out-of-hand' consumers, defined as those who ate at least $7 \mathrm{~g} / \mathrm{d}$ of nuts solely as nuts and not as part of other products, were studied because they were thought to make a more conscious decision to eat nuts. These consumers had higher intakes of energy, MUFA, PUFA, dietary fibre, $\mathrm{Cu}$ and $\mathrm{Mg}$ and lower intakes of carbohydrate, cholesterol and $\mathrm{Na}$ when compared with non-consumers. Similarly, King et al. ${ }^{(10)}$ reported that nuts

Abbreviations: 2008/09 NZANS, 2008/09 New Zealand Adult Nutrition Survey; NHANES, National Health and Nutrition Examination Survey; \%TE, percentage of total energy.

* Corresponding author: R. C. Brown, fax +64 3479 7958, email rachel.brown@otago.ac.nz 
made an important contribution to the diets of both nut consumers and nut snackers (individuals who consume nuts as snacks).

In support of these findings, intervention studies have reported improvements to nutrient profiles resulting from the addition of nuts to participants' diets without any other dietary advice. Increases in cis-unsaturated fat, vegetable protein, dietary fibre, vitamin $\mathrm{E}, \mathrm{Cu}$ and $\mathrm{Mg}$ have been observed ${ }^{(11-13)}$.

To date, no research has examined the nutrient contribution of nuts to the New Zealand diet, where dietary patterns may differ from countries where nut intake patterns have been previously described ${ }^{(7,9,10)}$. Traditionally, New Zealand diets have contained more meat and butter than that of other countries that are part of the Organisation for Economic Co-operation and Development, and, although declining over the last few decades, bread, potatoes and beef remain major sources of energy and nutrients ${ }^{(14)}$. This differs substantially from a Mediterranean eating pattern common to a number of European countries ${ }^{(15)}$. Compared with the USA, a greater percentage of energy is consumed from foods prepared at home in New Zealand, as opposed to fast food and restaurant food $^{(16)}$. Also, unlike in the USA, fortification of flour with folic acid is not mandatory, and fortification of foods with vitamin D is uncommon ${ }^{(17,18)}$. In addition, New Zealand soils are low in Se and I, meaning intakes of these micronutrients are relatively low ${ }^{(19)}$. Therefore, the aim of this study was to compare the nutrient profiles of nut consumers with that of non-nut consumers in a cross-section of the New Zealand population.

\section{Methods}

\section{Study population}

The 2008/09 New Zealand Adult Nutrition Survey (2008/09 NZANS) was a cross-sectional survey of New Zealanders aged 15 years and over, carried out from October 2008 to October 2009. A full description of the study design and methods is available elsewhere ${ }^{(20)}$, and only a summary is presented here. Participants were recruited using a three-stage process where 607 meshblocks were selected using a probability-proportionalto-size design. A meshblock is defined as a small geographical area within New Zealand defined by Statistics New Zealand. Each meshblock contains about 110 people in urban areas and sixty in rural areas. After random selection of a household, random selection of a participant within the household occurred. Oversampling of Māori and Pacific people and age groups 15-18 and 71 years was used in order to achieve adequate numbers for subgroup analyses by ethnicity and age.

Informed, written consent was obtained from each participant, or from the guardian of participants aged $<18$ years, before interviews. Ethical approval was gained from the New Zealand Health and Disability Multi-Region Ethics Committee (MEC/08/04/049). This study was conducted according to the guidelines laid down in the Declaration of Helsinki.

\section{Dietary assessment}

Survey data were collected at participants' homes by trained interviewers using computer-assisted personal interview software. An interviewer-administered multiple-pass 24-h diet recall method was used to collect quantitative information on all foods and drinks the participant consumed the previous day (from midnight to midnight). It included all foods and drinks consumed, both at and away from home.

In the first stage of the recall, a 'quick list' of all foods, beverages and dietary supplements consumed during the preceding day was obtained. In the second stage, detailed descriptions of all the foods and beverages consumed were collected. Information on any additions made to food before eating was also collected. In the third stage, estimates of the amounts of all foods and beverages consumed were obtained. The amount eaten was described by volume wherever possible (e.g. cups or tablespoons). In addition, food photographs, shape dimensions, food portion assessment aids (e.g. dried beans) and packaging information were used. Finally, in the fourth stage, the foods were reviewed and the information collected was checked. Any additions and changes were made at this point. Repeat interviews were conducted on 1180 participants, but these were not used here.

\section{Determination of nut consumption}

For the purpose of this analysis, the term 'nuts' included tree nuts, mixed nuts and peanuts. Chestnuts, coconut and coconut products were not included in this analysis as their nutrient profiles differ from the aforementioned 'nuts'. Tree nuts include almonds, Brazil nuts, cashews, hazelnuts, macadamias, mixed nuts, pecans, pine nuts, pistachios and walnuts. Nut intake was assessed using the 24-h recall data from the 2008/09 NZANS, and nut consumption was subsequently divided into three categories: (i) whole nuts including tree nuts, mixed nuts and peanuts eaten whole as part of a snack (e.g. mixed-nut snacks) or as an addition to a food/meal (e.g. almonds sprinkled on a salad); (ii) nut butters including those made from peanuts and tree nuts (e.g. peanut butter, hazelnut spread); and (iii) other sources including tree nuts, peanuts and mixed nuts eaten as ingredients in recipes or as part of commercial products (e.g. breakfast cereals, snack bars, satay). 'Total nuts' encompass whole nuts, nut butters and nuts from hidden sources. Participants who reported consuming zero quantity of any nuts in their 24-h recall were classified as 'non-nut consumers'. 'Whole nut consumers' were participants who reported consuming any amount of whole nuts, and 'total nut consumers' were participants who reported consuming any of whole nuts, nut butters and/or hidden sources of nuts.

\section{Demographic variables}

Demographic variables were selected a priori after reviewing the literature. Variables included sex, age category (15-18, 19-30, 31-50, 51-70, 71+ years), prioritised ethnicity, New Zealand Index of Deprivation (NZDep06), education, BMI and smoking status.

Ethnicity. Self-reported ethnicity was categorised into one of three ethnic groups based on a priority classification system 
using the coding prioritisation order (from highest to lowest) of Māori, Pacific and New Zealand European and Other.

New Zealand Index of Deprivation. NZDep06 is an area-based measure of deprivation, which uses nine variables from the New Zealand Census reflecting specified dimensions of both material and social deprivation. Each meshblock in New Zealand is given a score between 1 and 10, with a score of 1 reflecting the least deprived areas and 10 the most deprived. For the purpose of the 2008/09 NZANS, these scores were divided into quintiles where quintile 1 represents the $20 \%$ least deprived and quintile 5 the $20 \%$ most deprived areas.

Education. Participants were asked to report their highest school-level qualification and where appropriate their highest post-school qualification. Three groups comprising no formal school qualification, secondary school qualification only or post-school qualification (including trade certificates and university degrees) were used for these analyses.

Smoking status. Information was collected on smoking status during the interview, and participants were classified as a never smoker, ex-smoker or current smoker.

\section{Anthropometric measurements}

Trained interviewers carried out height and weight measurements in duplicate. Standing height was measured using a stadiometer (Seca 214) and weight using electronic scales (Tanita HD-351). BMI was calculated as weight $(\mathrm{kg}) /$ height squared $\left(\mathrm{m}^{2}\right)$. The WHO BMI cut-offs were used to categorise BMI status in participants aged 19 years and over (underweight: $<18.5 \mathrm{~kg} / \mathrm{m}^{2}$; normal range: $18.5-24.99 \mathrm{~kg} / \mathrm{m}^{2}$; overweight: $\geq 25-29.99 \mathrm{~kg} / \mathrm{m}^{2}$; obese: $\geq 30 \mathrm{~kg} / \mathrm{m}^{2}$ ). Relatively few individuals were classified as underweight ( $n$ 58), and this category was combined with the normal weight category. The Cole age- and sex-specific BMI cut-offs were used to categorise BMI status in those aged $15-18$ years $^{(21,22)}$

\section{Statistical analysis}

The complex survey design described above was accounted for in all analyses presented here. This includes incorporating both weights and clustering. The weights used were poststratification weights for the questionnaire component of the 2008/09 NZANS and are intended to reflect the New Zealand population aged 15 years and above.

Log transformations were made where this improved residual normality and/or homoscedasticity. Variables that were log transformed are presented as geometric means with differences reported as the percentage of difference between the geometric means. Unadjusted and adjusted differences for nutrient intakes between nut consumers and non-nut consumers are presented. Adjusted regression models included sex, age group, prioritised ethnicity, BMI category, NZDep06 quintile, education and smoking status. We also adjusted for energy intake in a third model for each intake outcome to determine the effects of nuts on nutrient intake, independent of energy. This is both to adjust for any potential confounding between micronutrient intakes and health by physical activity and metabolic efficiency (not measured in this study) not accounted for through including BMI in the models and to acknowledge that some nutrients are required in absolute amounts irrespective of energy, and others are required in relative amounts.

Stata Statistical Software 12.1 (StataCorp LP) was used for all statistical analyses. All statistical tests were two-sided and $P<0.05$ was considered statistically significant.

\section{Results}

\section{Characteristics of the sample}

Table 1 describes the characteristics of the 2008/09 NZANS sample and that of total and whole nut consumers. A total of 4721 participants were recruited and completed a 24-h diet recall.

\section{Nut intake}

We had previously described the nut intakes of New Zealanders ${ }^{(23)}$. In brief, the percentage of the population consuming whole nuts and total nuts on the day of the 24-h recall was $6.9 \%$ ( $n$ 240) and 28.9\% ( $n$ 1167), respectively. Among whole nut consumers, the mean portion size was $40(95 \%$ CI 33, 47) g/d for whole nuts, and, among consumers of any nut, the mean portion size was 18 (95\% CI 16, 20) g/d for total nuts.

\section{Nutrient intakes among whole nut consumers compared with non-whole nut consumers}

When adjusted for potential confounders (not including energy intake), reported energy intakes and total fat expressed both in absolute terms and as a percentage of total energy (\%TE) were significantly higher among whole nut consumers compared with non-whole nut consumers (all $P<0 \cdot 001$ ) (Table 2). When examining the different types of fat, SFA as \%TE was significantly lower among whole nut consumers compared with non-whole nut consumers $(P=0 \cdot 025)$. Conversely, both MUFA and PUFA when expressed in absolute amounts and as \%TE were significantly higher in whole nut consumers (all $P<0.001)$. Carbohydrate intakes expressed as \%TE $(P<0.001)$ were significantly lower among whole nut consumers, whereas protein intakes did not differ $(P=0 \cdot 165)$. Dietary fibre intake was significantly higher in whole nut consumers $(P<0 \cdot 001)$.

In terms of micronutrients, overall intakes were higher among whole nut consumers. When adjusted for potential confounders (not including energy intake), intakes of thiamin, riboflavin, niacin, vitamin $\mathrm{B}_{6}$, total folate, vitamin $\mathrm{C}$, vitamin $\mathrm{E}, \mathrm{Ca}, \mathrm{Cu}, \mathrm{Fe}$, $\mathrm{Mg}, \mathrm{P}, \mathrm{K}$, Se and $\mathrm{Zn}$ were all significantly higher among whole nut consumers compared with non-whole nut consumers (all $P<0 \cdot 011)$.

When intakes were further adjusted for energy intake, dietary cholesterol was significantly lower among whole nut consumers $(P<0.001)$, although dietary fibre remained significantly higher $(P<0 \cdot 001)$. Of the micronutrients, folate, vitamin $\mathrm{E}, \mathrm{Cu}, \mathrm{Mg}, \mathrm{P}, \mathrm{K}$ 
Table 1. Characteristics of survey participants in the 2008/09 New Zealand Adult Nutrition Survey (Numbers and percentages)

\begin{tabular}{|c|c|c|c|c|c|c|}
\hline \multirow[b]{2}{*}{ Demographic variables } & \multicolumn{2}{|c|}{ All survey participants } & \multicolumn{2}{|c|}{ Total nut consumers } & \multicolumn{2}{|c|}{ Whole nut consumers } \\
\hline & $n$ & Survey weighted (\%) & Survey weighted (\%) & $P^{*}$ & Survey weighted (\%) & $P \dagger$ \\
\hline Total population & 4721 & & & & & \\
\hline Sex & & & & 0.183 & & 0.351 \\
\hline Male & 2066 & $48 \cdot 6$ & $45 \cdot 4$ & & 43.7 & \\
\hline Female & 2655 & 51.4 & 54.6 & & 56.4 & \\
\hline Age (years) & & & & 0.005 & & $<0.001$ \\
\hline $15-18$ & 699 & $7 \cdot 0$ & 7.4 & & 1.8 & \\
\hline $19-30$ & 718 & $19 \cdot 7$ & 16 & & $10 \cdot 3$ & \\
\hline $31-50$ & 1344 & $36 \cdot 7$ & $42 \cdot 1$ & & 43 & \\
\hline $51-70$ & 895 & $27 \cdot 1$ & 25.9 & & $36 \cdot 3$ & \\
\hline $71+$ & 1065 & 9.6 & 8.6 & & $8 \cdot 7$ & \\
\hline Ethnicity & & & & $<0.001$ & & $<0.001$ \\
\hline NZEO & 2980 & $84 \cdot 3$ & 88.2 & & 93.6 & \\
\hline Māori & 1040 & $11 \cdot 1$ & $11 \cdot 3$ & & 4.9 & \\
\hline Pacific & 701 & 4.6 & 4.8 & & 1.5 & \\
\hline NZDep06 quintile & & & & $<0.001$ & & 0.032 \\
\hline Q1 (least deprived) & 664 & $20 \cdot 2$ & $21 \cdot 7$ & & 28.5 & \\
\hline Q2 & 829 & 21.4 & $24 \cdot 1$ & & $25 \cdot 1$ & \\
\hline Q3 & 761 & $21 \cdot 3$ & $24 \cdot 8$ & & 19.7 & \\
\hline Q4 & 1072 & $19 \cdot 0$ & 18.7 & & 14.9 & \\
\hline Q5 (most deprived) & 1395 & $18 \cdot 1$ & $10 \cdot 7$ & & 11.9 & \\
\hline Highest educational qualification & & & & $<0.001$ & & 0.023 \\
\hline No school qualification & 1217 & $18 \cdot 1$ & 13.9 & & $12 \cdot 0$ & \\
\hline School & 1413 & $26 \cdot 5$ & $25 \cdot 4$ & & $22 \cdot 6$ & \\
\hline Post-school & 2057 & 55.4 & $60 \cdot 7$ & & 65.5 & \\
\hline BMI $\left(\mathrm{kg} / \mathrm{m}^{2}\right)$ & & & & 0.010 & & 0.070 \\
\hline$<25$ & 1409 & 34.9 & 39.0 & & 44.4 & \\
\hline $25-29.9$ & 1581 & $37 \cdot 1$ & 37.9 & & 34.6 & \\
\hline$\geq 30$ & 1513 & $28 \cdot 0$ & $23 \cdot 1$ & & $21 \cdot 0$ & \\
\hline Smoking status & & & & $<0.001$ & & 0.013 \\
\hline Never smoked & 2393 & $50 \cdot 8$ & 59.9 & & $58 \cdot 8$ & \\
\hline Ex-smoker & 1274 & 26.5 & $27 \cdot 6$ & & $30 \cdot 3$ & \\
\hline Current smoker & 1074 & $22 \cdot 8$ & 12.5 & & $10 \cdot 9$ & \\
\hline
\end{tabular}

NZEO, New Zealand European and Other; NZDep06, New Zealand Index of Deprivation; Q, quintile.

* $P$ value for difference between all survey participants and total nut consumers.

t $P$ value for difference between all survey participants and whole nut consumers.

and $\mathrm{Zn}$ remained higher among whole nut consumers compared with non-whole nut consumers (all $P \leq 0 \cdot 044$ ). Conversely, vitamin $B_{12}$ intake was significantly lower among whole nut consumers $(P=0 \cdot 013)$.

\section{Nutrient intakes among total nut consumers compared with non-total nut consumers}

After adjustment for potential confounders (not including energy intake), mean reported energy intakes were significantly higher among total nut consumers compared with non-total nut consumers $(P<0.001)$ (Table 3$)$. When expressed in absolute terms and as \%TE, total fat, MUFA and PUFA were all significantly higher among total nut consumers (all $P<0.001)$. Intakes of SFA and carbohydrate were significantly higher in total nut consumers when expressed in absolute terms (all $P<0.001$ ) but not when expressed as \%TE (all $P \geq 0.082$ ). Intakes of dietary fibre were $27 \%$ higher in nut consumers compared with non-nut consumers $(P<0 \cdot 001)$.

For micronutrients, following adjustment for potential confounders (not including energy intake), the intakes of vitamin A, thiamin, riboflavin, niacin, vitamin $\mathrm{B}_{6}$, folate, vitamin $\mathrm{C}$, vitamin $\mathrm{E}, \mathrm{Ca}, \mathrm{Cu}, \mathrm{Fe}, \mathrm{Mg}, \mathrm{P}, \mathrm{K}$, Se and $\mathrm{Zn}$ were significantly higher among total nut consumers (all $P \leq 0 \cdot 009$ ).

When intakes were further adjusted for energy intake, dietary fibre remained significantly higher $(P<0.001)$ and dietary cholesterol significantly lower $(P<0.001)$ in total nut consumers compared with non-total nut consumers. Of the micronutrients, vitamin $\mathrm{E}, \mathrm{Ca}, \mathrm{Cu}, \mathrm{Fe}, \mathrm{Mg}$ and $\mathrm{P}$ remained statistically significantly higher among total nut consumers (all $P \leq 0 \cdot 007$ ).

\section{Contribution of whole nuts to daily energy and nutrient intakes}

When the study population was considered collectively, whole nuts contributed relatively minor amounts of energy and nutrients (Table 4). For example, whole nuts contributed only $0.7 \% \mathrm{TE}, 1.4 \%$ total fat (0.54\%TE), $0.76 \%$ SFA (0.08\%TE), $1.84 \%$ MUFA (0.28 \%TE), $2.04 \%$ PUFA ( $0.15 \%$ TE) and $0.68 \%$ of protein $(0.23 \% \mathrm{TE})$. When broken down by sex, these did not differ to a meaningful extent between men and women.

However, among whole nut consumers, whole nuts made a substantial contribution to energy and nutrient intakes. Whole nuts 
Table 2. Mean daily nutrient intake by consumption of whole nuts in the 2008/09 New Zealand Adult Nutrition Survey* (Mean values and $95 \%$ confidence intervals)

\begin{tabular}{|c|c|c|c|c|c|c|c|c|c|c|}
\hline \multirow[b]{2}{*}{ Nutrients } & \multicolumn{2}{|c|}{$\begin{array}{c}\text { Non-whole nut } \\
\text { consumers }(n 4481)\end{array}$} & \multicolumn{2}{|c|}{$\begin{array}{l}\text { Whole nut consumers } \\
(n 240)\end{array}$} & \multirow{2}{*}{$\begin{array}{l}\text { Unadjusted } \\
\text { difference }\end{array}$} & \multirow[b]{2}{*}{$95 \% \mathrm{Cl}$} & \multirow{2}{*}{$\begin{array}{l}\text { Unadjusted } \\
P\end{array}$} & \multirow{2}{*}{$\begin{array}{l}\text { Adjusted } \\
\text { difference }\end{array}$} & \multirow[b]{2}{*}{$95 \% \mathrm{Cl}$} & \multirow{2}{*}{$\begin{array}{c}\text { Adjusted } \\
\mathrm{P} \dagger\end{array}$} \\
\hline & Mean & $95 \% \mathrm{Cl}$ & Mean & $95 \% \mathrm{Cl}$ & & & & & & \\
\hline Energy (kJ) & 8160 & 7978,8346 & 9509 & 8878,10186 & $16 \cdot 5$ & $8 \cdot 4,25 \cdot 3$ & $<0.001$ & $20 \cdot 3$ & $12 \cdot 6,28 \cdot 6$ & $<0.001$ \\
\hline Protein $(\mathrm{g})$ & $77 \cdot 1$ & $75 \cdot 3,78 \cdot 9$ & $88 \cdot 1$ & $82 \cdot 0,94 \cdot 7$ & $14 \cdot 3$ & $5 \cdot 9,23 \cdot 3$ & 0.001 & $15 \cdot 3$ & $7 \cdot 4,23 \cdot 7$ & $<0.001$ \\
\hline Protein (\%TE) & $15 \cdot 8$ & $15 \cdot 3,16 \cdot 0$ & $15 \cdot 5$ & $14 \cdot 7,16 \cdot 3$ & -1.8 & $-7 \cdot 1,3 \cdot 7$ & 0.511 & $-4 \cdot 0$ & $-9 \cdot 4,1 \cdot 7$ & 0.165 \\
\hline Total fat (g) & $69 \cdot 7$ & $67 \cdot 8,71 \cdot 6$ & $90 \cdot 7$ & $83 \cdot 8,98 \cdot 1$ & $30 \cdot 1$ & $19 \cdot 7,41 \cdot 3$ & $<0.001$ & 35.4 & $25 \cdot 5,46 \cdot 2$ & $<0.001$ \\
\hline Total fat (\%TE) & 32.1 & $31 \cdot 7,32 \cdot 6$ & 35.9 & $34.5,37.5$ & 11.8 & $7 \cdot 1,16 \cdot 7$ & $<0.001$ & $12 \cdot 8$ & $8.0,17.9$ & $<0.001$ \\
\hline SFA $(g)$ & $26 \cdot 6$ & $25 \cdot 8,27 \cdot 4$ & $28 \cdot 1$ & $25 \cdot 5,30.9$ & 5.5 & $-4 \cdot 6,16 \cdot 6$ & 0.297 & $11 \cdot 3$ & $1 \cdot 3,22 \cdot 3$ & 0.027 \\
\hline SFA (\%TE) & 12 & $11 \cdot 8,12 \cdot 3$ & $10 \cdot 9$ & $10 \cdot 3,11 \cdot 6$ & $-9 \cdot 2$ & $-14 \cdot 7,-3 \cdot 3$ & 0.003 & $-7 \cdot 0$ & $-12.7,-0.9$ & 0.025 \\
\hline MUFA (g) & 24.8 & $24 \cdot 1,25 \cdot 6$ & 35.4 & $32 \cdot 7,38 \cdot 4$ & $42 \cdot 6$ & $31 \cdot 2,55 \cdot 0$ & $<0.001$ & 48.8 & $37 \cdot 8,60 \cdot 7$ & $<0.001$ \\
\hline MUFA (\%TE) & $11 \cdot 2$ & $11 \cdot 0,11 \cdot 4$ & $13 \cdot 8$ & $13 \cdot 1,14.5$ & $22 \cdot 8$ & $16 \cdot 5,29 \cdot 5$ & $<0.001$ & $24 \cdot 4$ & $17 \cdot 9,31 \cdot 2$ & $<0.001$ \\
\hline PUFA (g) & 9.4 & $9 \cdot 1,9 \cdot 7$ & $16 \cdot 5$ & $15 \cdot 0,1 \cdot 81$ & 75.4 & $59 \cdot 4,93 \cdot 1$ & $<0.001$ & $76 \cdot 3$ & $61 \cdot 0,93 \cdot 2$ & $<0.001$ \\
\hline PUFA (\%TE) & $4 \cdot 2$ & $4 \cdot 2,4 \cdot 3$ & $6 \cdot 4$ & $6 \cdot 0,6 \cdot 8$ & 51 & $41 \cdot 1,61 \cdot 3$ & $<0.001$ & $47 \cdot 3$ & $37 \cdot 8,57 \cdot 5$ & $<0.001$ \\
\hline Carbohydrate (g) & 223 & 218,229 & 234 & 214,257 & 4.9 & $-4 \cdot 7,15 \cdot 5$ & 0.327 & 7.8 & $-1.5,17.9$ & 0.102 \\
\hline Carbohydrate (\%TE) & $45 \cdot 7$ & $45 \cdot 2,46 \cdot 1$ & $41 \cdot 2$ & $38 \cdot 6,43.9$ & -9.9 & $-15 \cdot 4,-4 \cdot 0$ & 0.001 & $-10 \cdot 3$ & $-15 \cdot 4,-4 \cdot 6$ & 0.001 \\
\hline Sugars (g) & 95 & 92,98 & 108 & 98,119 & $14 \cdot 1$ & $2 \cdot 7,26 \cdot 7$ & 0.014 & $16 \cdot 7$ & $5 \cdot 2,29 \cdot 4$ & 0.004 \\
\hline Sugars (\%TE) & 18.5 & $18 \cdot 1,18 \cdot 9$ & $18 \cdot 1$ & $16 \cdot 8,19 \cdot 6$ & $-1 \cdot 8$ & $-9 \cdot 3,6 \cdot 2$ & 0.646 & $-2 \cdot 6$ & $-10 \cdot 2,5 \cdot 6$ & 0.526 \\
\hline Cholesterol (mg) & 208 & 200,216 & 179 & 150,213 & $-13 \cdot 9$ & $-28,2 \cdot 9$ & 0.099 & $-11 \cdot 1$ & $-25 \cdot 1,6 \cdot 4$ & 0.200 \\
\hline Total dietary fibre $(\mathrm{g})$ & $20 \cdot 2$ & $19.7,20.7$ & $28 \cdot 8$ & $26 \cdot 4,31 \cdot 3$ & $45 \cdot 5$ & $30 \cdot 4,55 \cdot 8$ & $<0.001$ & $37 \cdot 0$ & $25 \cdot 6,49 \cdot 4$ & $<0.001$ \\
\hline Vitamin A $(\mu \mathrm{g})$ & 647 & 624,670 & 728 & 625,748 & $12 \cdot 6$ & $-3 \cdot 8,31 \cdot 8$ & 0.138 & $9 \cdot 0$ & $-6 \cdot 8,27 \cdot 5$ & 0.280 \\
\hline Thiamin (mg) & $1 \cdot 15$ & $1 \cdot 12,1 \cdot 19$ & 1.35 & $1.23,1.48$ & $17 \cdot 4$ & $6 \cdot 6,29 \cdot 4$ & 0.001 & $17 \cdot 1$ & $6 \cdot 76,28 \cdot 4$ & 0.001 \\
\hline Riboflavin (mg) & 1.68 & $1.63,1.72$ & 1.9 & $1.73,2.09$ & $13 \cdot 4$ & $2 \cdot 9,25 \cdot 0$ & 0.011 & $12 \cdot 5$ & $2 \cdot 8,23 \cdot 1$ & 0.011 \\
\hline Niacin (mg) & $15 \cdot 3$ & $15 \cdot 1,16 \cdot 0$ & $18 \cdot 9$ & $17 \cdot 1,20 \cdot 8$ & 21.4 & $10 \cdot 0,34 \cdot 1$ & $<0.001$ & 23.4 & $12 \cdot 2,35 \cdot 6$ & $<0.001$ \\
\hline Vitamin $B_{6}(\mathrm{mg})$ & 1.52 & $1.48,1.57$ & 1.87 & $1.69,2.06$ & $22 \cdot 6$ & $10 \cdot 8,35 \cdot 7$ & $<0.001$ & $22 \cdot 4$ & $11 \cdot 1,34 \cdot 9$ & $<0.001$ \\
\hline Total folate $(\mu \mathrm{g})$ & 284 & 276,292 & 382 & 345,422 & 34.4 & $21 \cdot 2,49 \cdot 1$ & $<0.001$ & $30 \cdot 1$ & $17 \cdot 8,43 \cdot 7$ & $<0.001$ \\
\hline Vitamin $B_{12}(\mu \mathrm{g})$ & 2.99 & $2 \cdot 88,3.11$ & 2.93 & $2.54,3.37$ & $-2 \cdot 2$ & $-15 \cdot 3,12 \cdot 9$ & 0.759 & $-2 \cdot 1$ & $-15 \cdot 1,12 \cdot 9$ & 0.769 \\
\hline Vitamin C (mg) & 65.5 & $62 \cdot 4,68 \cdot 8$ & 95.9 & $82 \cdot 1,112 \cdot 1$ & $46 \cdot 4$ & $24 \cdot 5,72 \cdot 1$ & $<0.001$ & 31.8 & $11.4,55.9$ & 0.001 \\
\hline Vitamin E (mg) & 8.78 & $8.54,9.03$ & 14.45 & $13 \cdot 32,15 \cdot 66$ & 64.5 & $51 \cdot 1,79 \cdot 2$ & $<0.001$ & $61 \cdot 6$ & $48 \cdot 9,75 \cdot 2$ & $<0.001$ \\
\hline $\mathrm{Ca}(\mathrm{mg})$ & 721 & 701,74 & 859 & 774,953 & $19 \cdot 2$ & $7 \cdot 1,32 \cdot 7$ & 0.001 & $15 \cdot 8$ & $4 \cdot 4,28.5$ & 0.006 \\
\hline $\mathrm{Cu}(\mathrm{mg})$ & $1 \cdot 17$ & $1 \cdot 15,1 \cdot 20$ & 1.92 & $1.76,2.09$ & 63.5 & $49 \cdot 7,78 \cdot 6$ & $<0.001$ & 59.5 & $46.6,73.5$ & $<0.001$ \\
\hline $\mathrm{Fe}(\mathrm{mg})$ & $10 \cdot 27$ & $10.03,10.51$ & 12.85 & $11.78,14.03$ & $25 \cdot 2$ & $14.4,37 \cdot 0$ & $<0.001$ & $23 \cdot 6$ & $13 \cdot 6,34 \cdot 6$ & $<0.001$ \\
\hline $\mathrm{Mg}(\mathrm{mg})$ & 277 & 271,283 & 415 & 385,448 & 40 & $38 \cdot 7,62 \cdot 2$ & $<0.001$ & $45 \cdot 7$ & $35 \cdot 3,56 \cdot 9$ & $<0.001$ \\
\hline$P(\mathrm{mg})$ & 1250 & 1222, 1278 & 1571 & 1464,1685 & $25 \cdot 7$ & $16 \cdot 7,35 \cdot 3$ & $<0.001$ & 24.5 & $16 \cdot 2,33 \cdot 4$ & $<0.001$ \\
\hline $\mathrm{K}(\mathrm{mg})$ & 2790 & 2730,2852 & 3478 & 3240,3733 & $24 \cdot 6$ & $15 \cdot 8,34 \cdot 2$ & $<0.001$ & $21 \cdot 1$ & $13 \cdot 0,30 \cdot 2$ & $<0.001$ \\
\hline $\operatorname{Se}(\mu \mathrm{g})$ & 44.9 & $45 \cdot 4,46 \cdot 4$ & 55.9 & $50 \cdot 1,62 \cdot 4$ & $24 \cdot 6$ & $11 \cdot 2,39 \cdot 6$ & $<0.001$ & $25 \cdot 3$ & $11 \cdot 6,40 \cdot 7$ & $<0.001$ \\
\hline $\mathrm{Zn}(\mathrm{mg})$ & 9.53 & $9.31,9.76$ & 11.74 & $10 \cdot 75,12 \cdot 81$ & $23 \cdot 1$ & $12 \cdot 3,34 \cdot 9$ & $<0.001$ & $24 \cdot 2$ & $14.4,34.9$ & $<0.001$ \\
\hline
\end{tabular}

$\% \mathrm{TE}$, percentage of total energy.

* All variables were log transformed and geometric means are presented, with differences reported as the percentage difference between geometric means.

† Adjustment for age, sex, ethnicity, BMI, New Zealand Index of Deprivation, education and smoking status.

provided about $10 \% \mathrm{TE}$ intake for whole nut consumers. Whole nuts also provided about $20 \%$ of total fat intake for nut consumers, contributing about $8 \% \mathrm{TE}$. When examining the different types of fat, it can be seen that whole nuts supplied about $11 \%$ of SFA (contributing 3\%TE), with higher contributions of $27 \%$ (4\%TE) to MUFA and $30 \%$ (2.2\%TE) to PUFA. Whole nuts comprised approximately $10 \%$ of the protein intake of nut consumers, providing 3.4\%TE. When broken down by sex, these did not differ to a meaningful extent between men and women, especially when looked at as percentages of total intake.

\section{Discussion}

This was the first study to assess dietary intake in relation to nut consumption, in a cross-sectional survey of a population from the Southern Hemisphere, more specifically New Zealand. This study confirms the results from dietary surveys undertaken in the USA, which show nut consumption is associated with an improvement in nutrient intakes and overall diet quality.
Among nut consumers in New Zealand, nuts made a substantial contribution to TE, fat, MUFA, PUFA and dietary fibre. Nut consumers also had a more nutrient-dense diet in terms of micronutrients than did non-nut consumers.

We analysed the data both for whole nut consumers and total nut consumers. It has been previously postulated that 'outof-hand' nut consumers may differ from total nut consumers, in that they make a conscious decision to consume nuts, which may be associated with a desire for a healthier lifestyle ${ }^{(9)}$. This group is similar to the whole nut consumers in the present study. Given total nut consumption included nuts from other sources, which include confectionery and snacks that contain added sugar, fat and salt, consuming whole nuts might be expected to be associated with improved diet quality compared with other total nut consumers. We found that SFA and carbohydrate when expressed as \%TE were significantly lower for whole nut consumers compared with non-whole nut consumers, whereas this difference was not evident when comparing total nut consumers with non-nut consumers. Micronutrient intakes differed between nut consumers and 
Table 3. Mean daily nutrient intake by consumption of total nuts in the 2008/09 New Zealand Adult Nutrition Survey* (Mean values and $95 \%$ confidence intervals)

\begin{tabular}{|c|c|c|c|c|c|c|c|c|c|c|}
\hline \multirow[b]{2}{*}{ Nutrients } & \multicolumn{2}{|c|}{$\begin{array}{l}\text { Non-nut consumers } \\
(n \text { 3554) }\end{array}$} & \multicolumn{2}{|c|}{$\begin{array}{l}\text { Total nut consumers } \\
\qquad(n 1167)\end{array}$} & \multirow{2}{*}{$\begin{array}{l}\text { Unadjusted } \\
\text { difference }\end{array}$} & \multirow[b]{2}{*}{$95 \% \mathrm{Cl}$} & \multirow{2}{*}{$\begin{array}{c}\text { Unadjusted } \\
P\end{array}$} & \multirow{2}{*}{$\begin{array}{c}\text { Adjusted } \\
\text { difference† }\end{array}$} & \multirow[b]{2}{*}{$95 \% \mathrm{Cl}$} & \multirow{2}{*}{$\begin{array}{l}\text { Adjusted } \\
\text { P† }\end{array}$} \\
\hline & Mean & $95 \% \mathrm{Cl}$ & Mean & $95 \% \mathrm{Cl}$ & & & & & & \\
\hline Energy (kJ) & 7937 & 7735,8145 & 9058 & 875,9368 & $14 \cdot 1$ & $9.5,18.9$ & $<0.001$ & 16 & $11 \cdot 6,20 \cdot 6$ & $<0.001$ \\
\hline Protein $(\mathrm{g})$ & $75 \cdot 3$ & $73 \cdot 4,77 \cdot 3$ & 84.3 & $81 \cdot 2,87.5$ & $11 \cdot 8$ & $7 \cdot 0,16 \cdot 9$ & $<0.001$ & $12 \cdot 6$ & $8 \cdot 0,17 \cdot 3$ & $<0.001$ \\
\hline Protein (\%TE) & $15 \cdot 8$ & $15 \cdot 6,16 \cdot 1$ & $15 \cdot 5$ & $15 \cdot 2,15 \cdot 9$ & $-1 \cdot 8$ & $-4 \cdot 7,1 \cdot 1$ & 0.217 & $-2 \cdot 7$ & $-5 \cdot 6,0 \cdot 3$ & 0.078 \\
\hline Total fat (g) & $66 \cdot 9$ & $64 \cdot 9,69 \cdot 0$ & 82 & $78 \cdot 8,85.5$ & $22 \cdot 7$ & $16 \cdot 8,28 \cdot 8$ & $<0.001$ & $25 \cdot 3$ & $19 \cdot 8,31 \cdot 1$ & $<0.001$ \\
\hline Total fat (\%TE) & 31.7 & $31 \cdot 2,32 \cdot 2$ & $34 \cdot 1$ & $33.5,34.8$ & $7 \cdot 7$ & $5 \cdot 1,10 \cdot 4$ & $<0.001$ & 8.4 & $5 \cdot 7,11 \cdot 2$ & $<0.001$ \\
\hline SFA (g) & $25 \cdot 6$ & $24 \cdot 7,26 \cdot 5$ & $29 \cdot 6$ & $28 \cdot 3,31 \cdot 1$ & $15 \cdot 9$ & $9 \cdot 4,22 \cdot 7$ & $<0.001$ & 19 & $12 \cdot 7,25 \cdot 6$ & $<0.001$ \\
\hline SFA (\%TE) & 11.9 & $11 \cdot 6,12 \cdot 1$ & $12 \cdot 1$ & $11 \cdot 8,12 \cdot 5$ & $2 \cdot 0$ & $-1 \cdot 6,5 \cdot 7$ & 0.277 & 3.3 & $-0.4,7 \cdot 2$ & 0.082 \\
\hline MUFA (g) & 23.8 & $23 \cdot 1,24 \cdot 6$ & 29.9 & $28 \cdot 6,31 \cdot 3$ & $25 \cdot 6$ & $19 \cdot 3,32 \cdot 2$ & $<0.001$ & 28.4 & $22 \cdot 3,34 \cdot 7$ & $<0.001$ \\
\hline MUFA (\%TE) & $11 \cdot 1$ & $10 \cdot 8,11 \cdot 3$ & $12 \cdot 2$ & $11 \cdot 9,12 \cdot 5$ & $10 \cdot 5$ & $7 \cdot 2,14 \cdot 0$ & $<0.001$ & 11.5 & $8 \cdot 0,15 \cdot 6$ & $<0.001$ \\
\hline PUFA (g) & 8.8 & $8.6,9.1$ & $12 \cdot 5$ & $11 \cdot 9,13.1$ & 41.7 & $34.2,49 \cdot 4$ & $<0.001$ & 42 & $35 \cdot 0,49 \cdot 3$ & $<0.001$ \\
\hline PUFA (\%TE) & 4.1 & $4 \cdot 0,4 \cdot 2$ & $5 \cdot 1$ & $5 \cdot 0,5 \cdot 3$ & $24 \cdot 7$ & $20.5,29.1$ & $<0.001$ & 23.3 & $18 \cdot 9,27 \cdot 9$ & $<0.001$ \\
\hline Carbohydrate (g) & 216 & 211,222 & 245 & 236,254 & $13 \cdot 2$ & $8 \cdot 3,18 \cdot 4$ & $<0.001$ & 14 & $9.2,18.9$ & $<0.001$ \\
\hline Carbohydrate (\%TE) & $45 \cdot 4$ & $44 \cdot 9,46 \cdot 0$ & $45 \cdot 1$ & $44 \cdot 2,46 \cdot 1$ & -0.6 & $-3 \cdot 0,1 \cdot 8$ & 0.598 & -1.5 & $-3 \cdot 8,0 \cdot 8$ & 0.201 \\
\hline Sugars (g) & 90 & 87,94 & 109 & 105,115 & $21 \cdot 4$ & $14 \cdot 7,28 \cdot 3$ & $<0.001$ & $21 \cdot 6$ & $14 \cdot 9,28 \cdot 6$ & $<0.001$ \\
\hline Sugars (\%TE) & $18 \cdot 1$ & $17 \cdot 6,18 \cdot 7$ & $19 \cdot 4$ & $18 \cdot 7,20 \cdot 0$ & $6 \cdot 8$ & $2 \cdot 3,11 \cdot 5$ & 0.003 & 5.5 & $1 \cdot 0,10 \cdot 2$ & 0.017 \\
\hline Cholesterol (mg) & 208 & 199,217 & 199 & 185,214 & $-4 \cdot 4$ & $-11 \cdot 7,3 \cdot 5$ & 0.268 & -1.9 & $-9.5,6 \cdot 4$ & 0.649 \\
\hline Total dietary fibre $(\mathrm{g})$ & $19 \cdot 2$ & $18 \cdot 7,19.8$ & 24.9 & $23 \cdot 9,25 \cdot 9$ & $29 \cdot 6$ & $23.5,35.9$ & $<0.001$ & $26 \cdot 8$ & $21 \cdot 1,32 \cdot 8$ & $<0.001$ \\
\hline Vitamin A ( $\mu \mathrm{g})$ & 632 & 607,657 & 705 & 663,750 & $11 \cdot 7$ & $-3 \cdot 7,20 \cdot 2$ & 0.003 & $10 \cdot 5$ & $2 \cdot 6,19 \cdot 1$ & 0.009 \\
\hline Thiamin (mg) & 1.12 & $1.08,1 \cdot 16$ & 1.29 & $1.23,1.35$ & $15 \cdot 6$ & $9 \cdot 2,22 \cdot 4$ & $<0.001$ & $15 \cdot 7$ & $9 \cdot 3,22 \cdot 5$ & $<0.001$ \\
\hline Riboflavin (mg) & 1.64 & $1.59,1.69$ & 1.84 & $1.77,1.92$ & $12 \cdot 7$ & $7 \cdot 0,18 \cdot 6$ & $<0.001$ & 13.5 & $7 \cdot 9,19.3$ & $<0.001$ \\
\hline Niacin (mg) & $15 \cdot 2$ & $14 \cdot 8,15 \cdot 7$ & $17 \cdot 2$ & $16 \cdot 4,18 \cdot 0$ & $12 \cdot 9$ & $6 \cdot 7,19 \cdot 5$ & $<0.001$ & $13 \cdot 8$ & $7 \cdot 8,20 \cdot 1$ & $<0.001$ \\
\hline Vitamin $B_{6}(\mathrm{mg})$ & 1.49 & $1.44,1.55$ & 1.67 & $1.58,1.76$ & $11 \cdot 9$ & $4.9,19.4$ & 0.001 & 11.6 & $4 \cdot 8,18.9$ & 0.001 \\
\hline Total folate $(\mu \mathrm{g})$ & 276 & 267,285 & 327 & 311,343 & 18.5 & $11 \cdot 9,25 \cdot 6$ & $<0.001$ & $16 \cdot 2$ & $9 \cdot 7,23 \cdot 0$ & $<0.001$ \\
\hline Vitamin $B_{12}(\mu \mathrm{g})$ & 3.01 & $2 \cdot 88,3.15$ & 1.67 & $1.58,1.76$ & $-2 \cdot 8$ & $-10 \cdot 2,5 \cdot 2$ & 0.479 & 1.9 & $-9.5,6 \cdot 3$ & 0.636 \\
\hline Vitamin C (mg) & $62 \cdot 3$ & $58.8,65.9$ & 81.4 & $75 \cdot 1,88 \cdot 2$ & $30 \cdot 8$ & $18 \cdot 5,44 \cdot 3$ & $<0.001$ & $20 \cdot 6$ & $9 \cdot 1,33 \cdot 3$ & $<0.001$ \\
\hline Vitamin E (mg) & 8.33 & $8.07,8.59$ & 11.25 & $10 \cdot 78,11 \cdot 74$ & 35 & $28 \cdot 3,42 \cdot 0$ & $<0.001$ & $34 \cdot 3$ & $27 \cdot 9,41 \cdot 2$ & $<0.001$ \\
\hline $\mathrm{Ca}(\mathrm{mg})$ & 687 & 665,709 & 847 & 809,887 & 23.4 & $17 \cdot 0,30 \cdot 2$ & $<0.001$ & 21.8 & $15 \cdot 6,28 \cdot 2$ & $<0.001$ \\
\hline $\mathrm{Cu}(\mathrm{mg})$ & 1.11 & $1 \cdot 08,1 \cdot 14$ & 1.5 & $1.44,1.57$ & 35.5 & $29 \cdot 0,42 \cdot 3$ & $<0.001$ & 33.5 & $27 \cdot 2,40 \cdot 0$ & $<0.001$ \\
\hline $\mathrm{Fe}(\mathrm{mg})$ & 9.93 & $9.67,10 \cdot 20$ & $11 \cdot 76$ & $11 \cdot 29,12 \cdot 25$ & $18 \cdot 4$ & $12 \cdot 9,24 \cdot 2$ & $<0.001$ & $18 \cdot 1$ & $12 \cdot 9,23 \cdot 6$ & $<0.001$ \\
\hline $\mathrm{Mg}(\mathrm{mg})$ & 263 & 257,270 & 345 & 332,358 & 39.9 & $25 \cdot 4,36 \cdot 8$ & $<0.001$ & $29 \cdot 9$ & $24 \cdot 7,35 \cdot 3$ & $<0.001$ \\
\hline$P(m g)$ & 1205 & 1175,1236 & 1443 & 1393, 1493 & $19 \cdot 7$ & $14 \cdot 8,24 \cdot 7$ & $<0.001$ & 19.5 & $14 \cdot 9,24 \cdot 3$ & $<0.001$ \\
\hline $\mathrm{K}(\mathrm{mg})$ & 2719 & 2653,2787 & 3133 & 3020,3251 & $15 \cdot 2$ & $10 \cdot 3,20 \cdot 4$ & $<0.001$ & $14 \cdot 1$ & $9.5,18.9$ & $<0.001$ \\
\hline Se $(\mu \mathrm{g})$ & $44 \cdot 2$ & $42 \cdot 7,45 \cdot 9$ & 49 & $46 \cdot 5,51 \cdot 6$ & $10 \cdot 7$ & $4 \cdot 1,17 \cdot 7$ & 0.001 & 11.5 & $4.9,18.5$ & $<0.001$ \\
\hline $\mathrm{Zn}(\mathrm{mg})$ & 9.27 & $9.02,9.52$ & 10.74 & $10.31,11.19$ & $15 \cdot 9$ & $10 \cdot 4,21 \cdot 7$ & $<0.001$ & $16 \cdot 8$ & $11 \cdot 5,22 \cdot 3$ & $<0.001$ \\
\hline
\end{tabular}

$\% \mathrm{TE}$, percentage of total energy.

* All variables were log transformed and geometric means are presented, with differences reported as the percentage difference between geometric means. † Adjustment for age, sex, ethnicity, BMI, New Zealand Index of Deprivation, education and smoking status.

Table 4. Contribution of whole nuts to daily energy and nutrients overall and for whole nut consumers in the 2008/09 New Zealand Adult Nutrition Survey

(Mean values and $95 \%$ confidence intervals)

\begin{tabular}{|c|c|c|c|c|}
\hline \multirow[b]{2}{*}{ Contribution of nuts to energy and nutrients } & \multicolumn{2}{|c|}{ Total sample $(n 4721)$} & \multicolumn{2}{|c|}{ Whole nut consumers ( $n$ 240) } \\
\hline & Mean & $95 \% \mathrm{Cl}$ & Mean & $95 \% \mathrm{Cl}$ \\
\hline Energy (kJ) & 69 & 52,87 & 1007 & 832,1182 \\
\hline Total energy intake (\%) & 0.7 & $0.5,0.9$ & $10 \cdot 2$ & $8.5,11.9$ \\
\hline Total fat $(\mathrm{g})$ & 1.4 & $1 \cdot 1,1 \cdot 8$ & $20 \cdot 8$ & $17 \cdot 4,24.3$ \\
\hline Total fat intake (\%) & 1.4 & $1 \cdot 1,1.8$ & 21.0 & $18 \cdot 1,23.8$ \\
\hline Total energy from fat (\%) & 0.5 & $0.4,0.7$ & 7.8 & $6 \cdot 6,9 \cdot 1$ \\
\hline SFA (g) & 0.2 & $0.2,0.3$ & $3 \cdot 2$ & $2 \cdot 5,3 \cdot 8$ \\
\hline SFA intake (\%) & 0.8 & $0.6,1.0$ & 11.0 & $8 \cdot 8,13 \cdot 3$ \\
\hline Total energy from SFA (\%) & 0.1 & $0.1,0.1$ & 1.2 & $0.9,1.4$ \\
\hline MUFA (g) & 0.8 & $0.6,0.9$ & $10 \cdot 8$ & $9 \cdot 1,12 \cdot 6$ \\
\hline MUFA intake (\%) & 1.8 & $1 \cdot 4,2 \cdot 2$ & $26 \cdot 7$ & $23 \cdot 3,30 \cdot 0$ \\
\hline Energy from MUFA (\%) & 0.3 & $0.2,0.4$ & $4 \cdot 1$ & $3.4,4 \cdot 7$ \\
\hline PUFA (g) & 0.4 & $0.3,0.5$ & 5.8 & $4.8,6 \cdot 8$ \\
\hline PUFA intake (\%) & $2 \cdot 0$ & $1 \cdot 6,2 \cdot 5$ & $29 \cdot 7$ & $26 \cdot 1,33 \cdot 2$ \\
\hline Energy from PUFA (\%) & 0.2 & $0.1,0.2$ & $2 \cdot 2$ & $1 \cdot 8,2 \cdot 6$ \\
\hline Protein $(\mathrm{g})$ & 0.6 & $0.5,0.8$ & $9 \cdot 1$ & $7 \cdot 3,10 \cdot 9$ \\
\hline Protein intake (\%) & 0.7 & $0.5,0.9$ & 9.8 & $7 \cdot 9,11 \cdot 7$ \\
\hline Energy from protein (\%) & 0.2 & $0.2,0.3$ & 3.4 & $2 \cdot 7,4 \cdot 0$ \\
\hline
\end{tabular}


non-nut consumers for both whole nut and total nut intakes, although the differences were more pronounced for most nutrients among whole nut consumers.

Tree nuts and peanuts are rich sources of MUFA and PUFA, although low in SFA. This is reflected in the intakes of whole and total nut consumers, where the intake of unsaturated fats was significantly higher among nut consumers both in absolute terms and when expressed as \%TE intake. Conversely, when SFA was expressed as \%TE, intake was significantly lower in whole nut consumers. Intakes of dietary fibre were also higher among nut consumers compared with non-nut consumers. It is plausible that these intakes may account for much of the beneficial effects of nuts on cardiovascular health observed among nut consumers in both epidemiological ${ }^{(24-27)}$ and clinical studies ${ }^{(28-30)}$.

The 2008/09 NZANS reported that the mean contribution of SFA to daily energy intake among the New Zealand population was $13.1 \%$ for both males and females, which is above the acceptable macronutrient distribution range of $<10 \%{ }^{(31)}$. Conversely, the contribution to daily energy from both MUFA ( 12.4 and $13.3 \%$ for males and females, respectively) and PUFA ( 4.8 and $4.9 \%$ for males and females, respectively) was lower than that was reported for SFA. A heart-healthy dietary fatty acid profile promotes predominantly unsaturated fats, with low intakes of SFA. Given the fatty acid composition of nuts, and our data indicating a more favourable dietary fatty acid intake among nut consumers, increasing the nut intake of the general population is likely to result in positive effects on risk factors for chronic disease, in particular CVD.

Nut consumers also had significantly higher intakes of many micronutrients including thiamin, niacin, riboflavin, vitamin $\mathrm{B}_{6}$, folate, vitamin $\mathrm{C}$, vitamin $\mathrm{E}, \mathrm{Ca}, \mathrm{Cu}, \mathrm{Fe}, \mathrm{Mg}, \mathrm{P}, \mathrm{K}$, Se and $\mathrm{Zn}$. Of these folate, vitamin $\mathrm{E}, \mathrm{Cu}, \mathrm{Mg}, \mathrm{P}, \mathrm{K}$ and $\mathrm{Zn}$ remained significantly higher when additionally adjusted for energy intake. The nutrient compositions of different nuts vary, but particular nuts can be good sources of vitamin $\mathrm{E}$, folate, $\mathrm{Mg}, \mathrm{Ca}, \mathrm{Zn}, \mathrm{Cu}$ and Se. Therefore, the inclusion of nuts into the regular diet may improve the adequacy of intake of many of these essential micronutrients. It is important to note that for some of these nutrients the risk of inadequate intake among the New Zealand population was relatively high ${ }^{(31)}$. For example, the risk of inadequate intake of $\mathrm{Ca}$, Se and $\mathrm{Zn}$ was 59, 45 and $25 \%$, respectively. In addition, it was noted in the USA that tree nut consumers had higher intakes of 'shortfall' nutrients in the USA, including fibre, vitamin $\mathrm{E}, \mathrm{Ca}$ and $\mathrm{Mg}^{(7)}$. In addition, a recent analysis of the NHANES 2005-2010 cohort using usual intake data found that tree nut consumers were less likely to have inadequate intakes of vitamins $\mathrm{A}, \mathrm{E}$ and $\mathrm{C}$, folate, $\mathrm{Ca}, \mathrm{Fe}, \mathrm{Mg}$ and $\mathrm{Zn}$ compared with non-consumers ${ }^{(8)}$.

Our findings are in general agreement with that of other national nutrition surveys from the USA, which have reported greater intakes of unsaturated fat and a number of micronutrients among nut consumers in comparison with nonconsumers $^{(7,9,10)}$. Using NHANES 1999-2004 data, O'Neil et $a l .{ }^{(7)}$ reported that intakes of fibre, vitamins $\mathrm{A}, \mathrm{B}_{6}, \mathrm{C}, \mathrm{E}$ and $\mathrm{K}$, thiamin, riboflavin, folate, $\mathrm{Ca}, \mathrm{Mg}, \mathrm{P}, \mathrm{Fe}, \mathrm{Zn}, \mathrm{Cu}$ and $\mathrm{Mg}$ were higher, whereas the intake of $\mathrm{Na}$ was lower among tree nut consumers compared with non-consumers. Similarly, using data from the What We Eat in America/NHANES 2001-2004, King et $a l .{ }^{(10)}$ reported that, among individuals reporting consumption of nuts as snacks, nuts contributed between 25 and $35 \%$ of selected nutrients including fat, PUFA, MUFA, linoleic acid, Mg, $\mathrm{Cu}$ and vitamin E. This is similar to our study where whole nuts contributed $27 \%$ (4\%TE) to MUFA and $30 \%$ (2.2\%TE) to PUFA intake. King et al. ${ }^{(10)}$ also identified nutrients and food components that were consumed in significantly higher quantities by nut consumers compared with non-nut consumers, namely vitamins $\mathrm{E}$ and $\mathrm{K}$, folate, $\beta$-carotene, lutein, zeaxanthin, $\mathrm{Mg}, \mathrm{P}, \mathrm{Cu}$, Se, $\mathrm{K}$ and $\mathrm{Zn}$. Conversely, vitamin $\mathrm{B}_{12}$, retinol, lycopene and $\mathrm{Na}$ were consumed in significantly lower amounts by nut consumers ${ }^{(10)}$. In our study, vitamin $\mathrm{B}_{12}$ was the only nutrient consumed in significantly lower quantities among nut consumers. It is possible that this may reflect the higher rates of nut consumption among vegetarians and vegans.

The results of the present study should be interpreted with several limitations in mind. First, the cross-sectional design of the study means that we cannot draw causal inferences. Although a better diet quality was observed among nut consumers compared with non-nut consumers in our study and others due to the addition of nutrient-dense nuts to the diet, an alternative explanation is that nut consumption may be a marker of a better diet overall or an indicator of particular dietary patterns (such as vegetarianism). Thus, residual confounding where a healthier lifestyle may mediate the association of nuts with diet quality and health outcomes cannot be excluded. Although BMI and smoking status were collected as part of the 2008/09 NZANS, measures of physical activity were not. Nut consumers may simply be more health conscious than non-nut consumers. In addition, dietary intake included only one 24-h diet recall for the majority of participants and therefore may not represent usual nut intake. Under (and potentially over)-reporting can also be an issue given that diet was selfreported. We were unable to discriminate between underreporters and under-consumers; thus, all participants were included in the analysis. However, rigorous coding of food items collected through a multi-stage process and the use of New Zealand-specific food composition data allow confidence in the collected estimates of intake for both nuts and nutrients. In addition, 24-h recalls yield reasonably accurate group estimates of nutrient intake ${ }^{(32)}$. Even if there are biases leading to under- or over-reporting of total dietary intakes, there are no reasons to suspect these would affect the associations reported here. Other strengths of the study include its large sample size, permitting precise estimation of effects, and the use of a representative, after weighting, population-based sample.

\section{Conclusions}

This is the first study using national data from a population in the Southern Hemisphere, more specifically New Zealand, to examine the effects of nut consumption on dietary intake. In agreement with the results of other studies conducted in the USA, nut consumption was associated with improved nutrient profiles, especially an increase in unsaturated fats and a number of essential vitamins and minerals, which could collectively reduce the risk for chronic disease, in particular for CVD. 


\section{Acknowledgements}

The authors thank the 4721 New Zealanders who participated in the 2008/09 New Zealand Adult Nutrition Survey.

The New Zealand Ministry of Health funded the 2008/09 New Zealand Adult Nutrition Survey. The New Zealand Crown is the owner of the copyright for the survey data. The results presented in this paper are the work of the authors.

The authors' contributions were as follows: R. C. B., W. P., A. R. G. and E. F. designed and/or conducted the research; R. C. B. performed the statistical analyses with assistance from A. R. G.; C. S., E. F. and R. C. B. managed the dietary analysis; W. P. was the principal investigator of the 2008/09 New Zealand Adult Nutrition Survey; R. C. B., S. L. T., A. R. G. and A. C. wrote the manuscript; all authors reviewed and approved the final manuscript.

There are no conflicts of interest.

\section{References}

1. Kris-Etherton PM, Yu-Poth S, Sabate J, et al. (1999) Nuts and their bioactive constituents: effects on serum lipids and other factors that affect disease risk. Am J Clin Nutr 70, 504S-511S.

2. Nash SD \& Nash DT (2008) Nuts as part of a healthy cardiovascular diet. Curr Atheroscler Rep 10, 529-535.

3. Ros E (2010) Health benefits of nut consumption. Nutrients $\mathbf{2}$, 652-682.

4. Alasalvar C \& Bolling BW (2015) Review of nut phytochemicals, fat-soluble bioactives, antioxidant components and health effects. Br J Nutr 113, S68-S78.

5. Brufau G, Boatella J \& Rafecas M (2006) Nuts: source of energy and macronutrients. Br J Nutr 96, S24-S28.

6. Bolling BW, Chen CY, McKay DL, et al. (2011) Tree nut phytochemicals: composition, antioxidant capacity, bioactivity, impact factors. A systematic review of almonds, Brazils, cashews, hazelnuts, macadamias, pecans, pine nuts, pistachios and walnuts. Nutr Res Rev 24, 244-275.

7. O'Neil CE, Keast DR, Fulgoni VL, et al. (2010) Tree nut consumption improves nutrient intake and diet quality in US adults: an analysis of National Health and Nutrition Examination Survey (NHANES) 1999-2004. Asia Pac J Clin Nutr 19, 142-150.

8. O'Neil CE, Nicklas TA \& Fulgoni VL 3rd (2015) Tree nut consumption is associated with better nutrient adequacy and diet quality in adults: National Health and Nutrition Examination Survey 2005-2010. Nutrients 7, 595-607.

9. O'Neil CE, Keast DR, Nicklas TA, et al. (2012) Out-of-hand nut consumption is associated with improved nutrient intake and health risk markers in US children and adults: National Health and Nutrition Examination Survey 1999-2004. Nutr Res 32, 185-194.

10. King JC, Blumberg J, Ingwersen L, et al. (2008) Tree nuts and peanuts as components of a healthy diet. J Nutr $\mathbf{1 3 8}$, 1736S-1740S.

11. Griel AE, Eissenstat B, Juturu V, et al. (2004) Improved diet quality with peanut consumption. J Am Coll Nutr 23, 660-668.

12. Jaceldo-Siegl K, Joan S, Rajaram S, et al. (2004) Long-term almond supplementation without advice on food replacement induces favourable nutrient modifications to the habitual diets of free-living individuals. BrJ Nutr 92, 533-540.
13. Tey SL, Brown R, Gray A, et al. (2011) Nuts improve diet quality compared to other energy-dense snacks while maintaining body weight. J Nutr Metab 2011, 357350.

14. Smith C, Gray AR, Mainvil LA, et al. (2015) Secular changes in intakes of foods among New Zealand adults from 1997 to 2008/09. Public Health Nutr, 1-11.

15. Willett WC, Sacks F, Trichopoulou A, et al. (1995) Mediterranean diet pyramid: a cultural model for healthy eating. Am J Clin Nutr 61, 1402S-1406S.

16. Smith C, Gray AR, Fleming EA, et al. (2014) Characteristics of fast-food/takeaway-food and restaurant/cafe-food consumers among New Zealand adults. Public Health Nutr 17, 2368-2377.

17. Crider KS, Bailey LB \& Berry RJ (2011) Folic acid food fortification - its history, effect, concerns, and future directions. Nutrients 3, 370-384.

18. Nowson CA, McGrath JJ, Ebeling PR, et al. (2012) Vitamin D and health in adults in Australia and New Zealand: a position statement. Med J Aust 196, 686-687.

19. Thomson CD (2004) Selenium and iodine intakes and status in New Zealand and Australia. Br J Nutr 91, 661-672.

20. University of Otago and Ministry of Health (2011) Methodology Report for the 2008/09 New Zealand Adult Nutrition Survey. Wellington: Ministry of Health.

21. Cole TJ, Bellizzi MC, Flegal KM, et al. (2000) Establishing a standard definition for child overweight and obesity worldwide: international survey. BMJ 320, 1240-1243.

22. Cole TJ, Flegal KM, Nicholls D, et al. (2007) Body mass index cut offs to define thinness in children and adolescents: international survey. BMJ 335, 194-197.

23. Brown RC, Tey SL, Gray AR, et al. (2014) Patterns and predictors of nut consumption: results from the 2008/09 New Zealand Adult Nutrition Survey. Br J Nutr 112, 2028-2040.

24. Bao Y, Han J, Hu FB, et al. (2013) Association of nut consumption with total and cause-specific mortality. $N$ Engl J Med 369, 2001-2011.

25. Hshieh TT, Petrone AB, Gaziano JM, et al. (2015) Nut consumption and risk of mortality in the Physicians' Health Study. Am J Clin Nutr 101, 407-412.

26. Luo C, Zhang Y, Ding YS, et al. (2014) Nut consumption and risk of type 2 diabetes, cardiovascular disease, and all-cause mortality: a systematic review and meta-analysis. Am J Clin Nutr 100, 256-269.

27. Zhou DH, Yu HB, He F, et al. (2014) Nut consumption in relation to cardiovascular disease risk and type 2 diabetes: a systematic review and meta-analysis of prospective studies. Am J Clin Nutr 100, 270-277.

28. Blanco Mejia S, Kendall CW, Viguiliouk E, et al. (2014) Effect of tree nuts on metabolic syndrome criteria: a systematic review and metaanalysis of randomised controlled trials. BMJ Open 4, e004660.

29. Sabate J, Oda K \& Ros E (2010) Nut consumption and blood lipid levels: a pooled analysis of 25 intervention trials. Arch Intern Med 170, 821-827.

30. Viguiliouk E, Kendall CW, Blanco Mejia S, et al. (2014) Effect of tree nuts on glycemic control in diabetes: a systematic review and meta-analysis of randomized controlled dietary trials. PLOS ONE 9, e103376.

31. University of Otago and Ministry of Health (2011) A Focus on Nutrition: Key Findings of the 2008/09 New Zealand Adult Nutrition Survey. Wellington: Ministry of Health.

32. Thompson FE \& Byers T (1994) Dietary assessment resource manual. J Nutr 124, 2245S-2317S. 